\title{
Laboreal
}

Volume $6 \mathrm{~N}^{\circ} 2$ | 2010

Varia

\section{INSAT2010 - Inquérito Saúde e Trabalho : outras questões, novas relações}

INSAT2010 - Encuesta Salud Y Trabajo: otras cuestiones, nuevas relaciones INSAT2010 - Enquête Santé et Travail : autres questions, nouvelles relations INSAT2010 - Health and Work Inquiry: other questions, new relationships

\section{Carla Barros-Duarte e Liliana Cunha}

\section{(2) OpenEdition}

\section{Journals}

\section{Edição electrónica}

URL: http://journals.openedition.org/laboreal/8700

DOI: $10.4000 /$ laboreal. 8700

ISSN: 1646-5237

\section{Editora}

Universidade do Porto

\section{Refêrencia eletrónica}

Carla Barros-Duarte e Liliana Cunha, «INSAT2010 - Inquérito Saúde e Trabalho : outras questões, novas relações », Laboreal [Online], Volume 6 N² | 2010, posto online no dia 01 dezembro 2010 consultado o 10 outubro 2019. URL : http://journals.openedition.org/laboreal/8700 ; DOI : 10.4000/ laboreal.8700

Este documento foi criado de forma automática no dia 10 outubro 2019.

Laboreal está licenciado com uma Licença Creative Commons - Atribuição-NãoComercial 4.0 Internacional. 


\title{
INSAT2010 - Inquérito Saúde e Trabalho : outras questões, novas relações
}

\author{
INSAT2010 - Encuesta Salud Y Trabajo : otras cuestiones, nuevas relaciones \\ INSAT2010 - Enquête Santé et Travail : autres questions, nouvelles relations \\ INSAT2010 - Health and Work Inquiry: other questions, new relationships
}

\section{Carla Barros-Duarte e Liliana Cunha}

\section{NOTA DO EDITOR}

Manuscrito recebido em : Outubro/2010

Aceite após peritagem em : Dezembro/2010

\section{NOTA DO AUTOR}

O que fazer para ter acesso ao inquérito INSAT2010?

Para ter acesso ao inquérito INSAT é fundamental que faça o pedido escrevendo uma mensagem electrónica às autoras do artigo (cujos endereços electrónicos estão indicados no início do artigo). No conteúdo desta mensagem devem figurar alguns dados pessoais (nomeadamente, o nome, a afiliação profissional, ...), bem como as razões que explicam o facto de considerar útil ter acesso a este instrumento. Com base na justificação deste pedido, será ponderado o envio do inquérito.

Esta opção visa evitar possíveis utilizações abusivas do inquérito. Mas também visa facilitar o acesso das autoras às experiências desenvolvidas a partir do instrumento $e$ ampliar a "equipa de investigação" que tem vindo a utilizá-lo nas suas pesquisas. Por estas razões, qualquer novo utilizador deverá fazer o seu pedido, sendo assim esperado que seja evitada a partilha do instrumento com utilizadores desconhecidos. No caso de se tratar de projectos desenvolvidos no Brasil, o pedido pode ser dirigido 
directamente à Prof. Doutora Jussara Cruz de Brito (Fiocruz) - jussara@ensp.fiocruz.br - e à Prof. Doutora Simone Oliveira - simone@ensp.fiocruz.br.

\section{Introdução}

1 A apresentação de uma versão actualizada do INSAT - Inquérito Saúde e Trabalho que, em 2007, se anunciou como uma "proposta metodológica para a análise dos efeitos das condições de trabalho na saúde" (Barros-Duarte, Cunha \& Lacomblez, 2007) vem confirmar a experiência positiva de se ter proposto um instrumento neste domínio: suficientemente global - que permitisse recolher de forma completa os factores que intervêm nas várias dimensões de saúde - e suficientemente específico - que abrangesse os outros problemas e doenças provocadas pelas condições de trabalho, para além das doenças profissionais oficialmente reconhecidas.

2 As mudanças que se fazem sentir ao nível das condições de trabalho e de emprego, tornadas visíveis, nomeadamente, através dos últimos inquéritos europeus sobre as condições de trabalho [1] e os contributos associados à utilização do INSAT, em diferentes sectores de actividade, sustentaram o interesse de criar um espaço de análise e reflexão de questões, levantadas entre outros pelas novas relações entre saúde e trabalho, e perspectivar deste modo uma evolução deste instrumento.

\section{Balanço das experiências de utilização do INSAT}

Desde que foi construído o INSAT e publicado o artigo que o apresenta (Barros-Duarte, Cunha \& Lacomblez, 2007) demos início à sua utilização em diferentes sectores de actividade e recebemos também alguns pedidos de investigadores/técnicos da área da saúde que se interessam pela problemática de utilização do inquérito, o que acabou por contribuir para o alargamento dos domínios e dos contextos da sua aplicação. Até este momento, o INSAT foi já aplicado no sector dos transportes rodoviários colectivos de passageiros $(n=160)$; no sector da educação $(n=30)$ e formação $(n=70)$; na Administração local - serviços municipalizados de água e saneamento $(\mathrm{n}=400)$; nas forças policiais $(\mathrm{n}=12)$ (pesquisa ainda em curso); e no sector da indústria, em diferentes áreas de actividade $(\mathrm{n}=239)$. Também no Brasil, no âmbito das pesquisas de uma equipa da Fiocruz [²], está em experimentação uma versão adaptada, não só às especificidades linguísticas do português tal como praticado no país, como ainda a algumas particularidades de actividades ditas "de serviço".

4 Os dados conseguidos apresentam obviamente limitações, particularmente se tivermos a pretensão de avançar em tratamentos estatísticos suficientemente elaborados para legitimar conclusões convincentes.

5 Contudo, a experiência adquirida permitiu desenvolver, ao mesmo tempo, o trabalho de aperfeiçoamento do instrumento, a ponderação de certas opções metodológicas e a clarificação do debate que pretendemos convocar com o INSAT - cujo estatuto híbrido obriga a situar-se entre as ambições dos grandes números e a "modéstia" do estudo de caso.

6 Neste sentido, foi determinante a partilha com os diferentes investigadores, fornecendo-nos as suas dúvidas e seus comentários críticos, sustentados nas experimentações do instrumento e na especificidade dos terrenos em análise. Grande 
parte das alterações introduzidas no INSAT2007 responderam assim a limitações observadas durante a sua aplicação ou a aspectos que não foram devidamente considerados.

7 Podemos sintetizar as alterações efectuadas na versão de 2007 do inquérito referindo três focos de análise : os contextos de aplicação do inquérito (o sector de actividade) ; os diferentes usos possíveis (opções metodológicas de aplicação do inquérito); as diferentes perspectivas na utilização dos dados, nomeadamente consoante a abordagem privilegiada pelo investigador/interventor - sejam psicólogos do trabalho, médicos, ergónomos, técnicos de segurança).

8 Ilustramos a seguir, com alguns exemplos significativos e concretos, a introdução de algumas novas questões, ou a reformulação de existentes, que caracterizam esta nova versão do INSAT :

\subsection{Contextos de aplicação do INSAT}

A referência ao tempo de trabalho é ampliada nesta versão do INSAT :

I. Na versão de 2007 do inquérito era pedido aos trabalhadores que identificassem qual o horário de trabalho que praticam ou praticaram. Tratava-se de uma questão enquadrada na mera caracterização da situação de trabalho. Não obstante, a pesquisa no sector dos transportes rodoviários de passageiros, onde o trabalho se encontra frequentemente organizado em horários atípicos, tornou relevante o reconhecimento desta questão como fonte de constrangimento, nomeadamente, quando não se tem qualquer possibilidade de alterar este horário. Foram, então, identificados diferentes tipos de horários e especificado o seu significado (por exemplo, "horário irregular");

II. Embora muitos trabalhadores assinalem trabalhar em horários irregulares e conseguirem, apesar disso, conciliar a vida de trabalho com a vida fora do trabalho não eram dadas condições para que expressassem o incómodo que isto pode suscitar. Ora, os estudos que temos vindo a desenvolver no sector dos transportes têm confirmado o custo para a saúde das tentativas, quase permanentes, dos ajustamentos entre as exigências do trabalho e da vida fora do trabalho (Cunha, Nogueira \& Lacomblez, forthcoming). Neste sentido, foram introduzidas questões complementares na análise das relações entre a vida de trabalho e a vida fora do trabalho, abordando o facto de a conciliação ser ou não possível, mas também os custos que acaba por comportar um tal compromisso ;

III. Para além da referência ao tempo de trabalho, traduzido por Quéinnec (2007) como relativo às durações e horários da actividade profissional, foram introduzidas nesta versão do inquérito questões alusivas ao tempo do trabalho, entendido como a delimitação entre a "temporalidade profissional e a temporalidade sócio-familiar da vida fora do trabalho" (idem, p. 90, tradução livre). Uma dessas questões diz respeito precisamente à temporalidade da interface entre a vida de trabalho e a vida fora do trabalho: o tempo despendido nas deslocações de e para o trabalho, que alguns trabalhadores haviam já assinalado como um custo associado à actividade de trabalho.

\subsection{Aspectos metodológicos}

I. A referência no INSAT à "exposição actual" e à "exposição no passado" relativamente a determinadas características do trabalho foi, no período de aplicação do inquérito, duplamente questionada : sobre, por um lado, o que designa "trabalho passado" e, por outro lado, sobre a ausência de medida da duração da exposição. Assumimos, então, nesta nova versão que o trabalho passado se poderia reportar a uma situação vivida noutro local de 
trabalho ou, no mesmo local, ainda que relativamente a outras funções. Acrescentamos também a opção de as pessoas assinalarem, em simultâneo, que se encontram expostas "no trabalho actual" e "no trabalho passado". Relativamente à inexistência de uma medida da duração da exposição, tal pode ser justificado pelo facto de isso condicionar a referência a actividades ou postos de trabalho ocupados por um curto prazo de tempo, mas também porque a duração da exposição não pode, por si mesma, ser encarada como a causa de determinados problemas de saúde. É na interacção entre as diferentes exposições que podemos encontrar, muitas vezes, as razões associadas a certas queixas relacionadas com o estado de saúde.

II. A utilização do INSAT como um instrumento de reflexão esteve na base da opção de pedir, num primeiro momento, ao trabalhador que dissesse de forma mais objectiva a que condições de trabalho se encontra exposto (e.g. ruído, vibrações, situações de hipersolicitação) e de solicitar, mais tarde, que se pronunciasse, de forma mais subjectiva, ao grau de incómodo que, para si, essas condições de trabalho provocam. Confrontados com esta sequência nas questões colocadas, alguns trabalhadores comentaram que já haviam respondido e que se tratava de uma questão "repetida". Na verdade, o que procurávamos era criar condições para uma evolução na reflexão do sujeito acerca do seu trabalho e do seu impacto na saúde. Neste sentido, optámos pela introdução da escala do incómodo ao mesmo tempo em que se abordam as questões da "exposição", admitindo que estas são questões reflectidas em simultâneo - aliás, é pelo grau de incómodo que a exposição a determinadas características do trabalho gera, que são reconhecidas como factores de risco.

\subsection{Outras características da saúde}

I. Na avaliação da percepção do estado de saúde, introduziu-se nesta nova versão do inquérito um instrumento que, centrado na opinião do sujeito, constitui uma base de informação orientada para a (auto)apreciação do estado de saúde. Recorreu-se para o efeito ao Perfil de Saúde de Nottingham (NHP) [3], já por nós experimentado no âmbito de questionários mais abrangentes (nomeadamente, "Inquérito SIT - Saúde, Idade e Trabalho") ["]. Na verdade, é considerado um dos melhores instrumentos preditores da saúde (VISAT, 1994 ; Derriennic, Touranchet, \& Volkoff, 1996), sendo constituído por um conjunto de itens agrupados em seis dimensões de saúde, designadamente : a energia, a dor, as reacções emocionais, o sono, o isolamento social e a mobilidade física.

\section{Uma abordagem metodológica multi-nível : reforçar os pontos de ancoragem do INSAT}

Dar visibilidade à evolução das formas de organização do trabalho e aos novos factores de risco que se colocam em contexto de trabalho é uma das características que deve estar presente em todos os instrumentos que pretendem avaliar as relações entre o trabalho e a saúde. A especificidade do Inquérito INSAT foi adoptar uma abordagem que tem como objectivo "compreender de que forma os trabalhadores avaliam as características e as condições do seu trabalho, o seu estado de saúde, e que tipo de relações estabelecem entre a sua saúde e o seu trabalho" (Barros-Duarte, Cunha, Lacomblez, 2007, p. 59).

11 O Inquérito INSAT, cuja lógica de base se enquadra numa tradição de certos estudos epidemiológicos (Derriennic, Touranchet \& Volkoff, 1996 ; Cassou et al., 2001 ; Molinié \& Volkoff, 2002), foi construído de modo a responder à complexidade do estudo das relações entre trabalho e saúde: integrou, quer na sua concepção quer na sua 
formulação, conhecimentos provenientes de disciplinas como a demografia ou a medicina do trabalho; mas considerou, ainda, o que realça uma longa tradição de estudos de terreno, mais qualitativos, desenvolvidos nomeadamente pela ergonomia ou pela psicologia do trabalho, e que contribuíram para um melhor entendimento da variabilidade e da diversidade das relações entre a saúde e o trabalho.

Nesta perspectiva, pretendemos inserir o INSAT em alguns dos modelos mais recentes de epidemiologia que, ao contrário dos modelos de epidemiologia de inspiração mais "positivista" - que, tendencialmente, se baseiam no estudo da relação directa e unívoca entre uma causa e uma patologia específica - integram outros factores, nomeadamente as relações entre as características profissionais e as condições de trabalho, os factores extra-profissionais e as condições de vida, abarcando, igual, e deliberadamente, as queixas e os problemas ditos "infra-patológicos" nas suas relações com o trabalho.

Trata-se, portanto, de uma abordagem metodológica quantitativa, mas como realçou Volkoff (2010) pode encontrar o seu espaço de análise e investigação em abordagens mais "compreensivas" do que "explicativas" e que, desde a concepção à análise e interpretação dos resultados, assume uma combinação coerente entre a análise da actividade e o inquérito por questionário.

De facto, a complexidade que caracteriza as relações entre saúde e trabalho envolvem a compreensão de um conjunto de factores que interagem mutuamente e a diferentes níveis da vida do indivíduo, solicitando assim, uma abordagem global e multifacetada, sustentada em quatro pontos de ancoragem que o INSAT procura agora reforçar.

Os quatro pontos de ancoragem de seguida apresentados reflectem já as preocupações que estiveram na origem da concepção do INSAT2007 e que, ao longo destes três anos, foram melhor sustentados na versão do INSAT2010 :

- integração permanente do conhecimento proveniente dos estudos de terreno, de modo a caracterizar e compreender o contexto de trabalho (3.1);

- consideração de todos os efeitos do trabalho na saúde (patologias e infra-patologias), de modo a incorporar a dimensão subjectiva dos efeitos do trabalho na saúde (3.2);

- assumpção de uma perspectiva centrada na pessoa, de modo a facilitar a expressão e a explicitação do vivido no trabalho (3.3) ;

- focalização numa análise integrada dos resultados, que procura desenvolver uma "estatística aberta» (3.4).

\subsection{Caracterizar e compreender o contexto de trabalho}

O INSAT constitui um bom exemplo de um instrumento de avaliação suficientemente atento à diversidade e variabilidade das questões que se colocam em contexto de trabalho. Através do discurso e das vivências no trabalho, outras questões emergem no estudo da saúde ocupacional e ganham sentido e consistência se colocarmos no centro da análise o contexto de trabalho e, mais concretamente, a actividade de trabalho.

Neste sentido, a actividade passa a ser o elemento central e estruturante do contexto de trabalho, lugar de integração dos constrangimentos do trabalho, por um lado, e da saúde e do bem-estar, por outro lado. Compreender a actividade de trabalho é compreender a actividade humana, que no contexto profissional é traduzida em comportamentos e condutas humanas que não se esgotam no mero cumprimento da tarefa. 
18 Na verdade, é a actividade que determina o conjunto de todas as acções (pensamentos, sentimentos e emoções) que caracterizam o comportamento humano no trabalho e que, inevitavelmente, interferem na construção da saúde e na forma como são percebidas e explicitadas estas relações. As dimensões pessoal e social da actividade de trabalho já integradas no INSAT2007 são agora reforçadas pela incorporação de outros factores psicossociais, como as novas formas de relação salarial e de horários, a conciliação entre a vida profissional e a vida privada, entre outros, que, aparentemente mais afastados da caracterização da actividade de trabalho, permitem compreender, também, a dimensão emocional que lhe está associada (Davezies, 2009).

De facto, a integração de um conjunto diverso de questões relativas ao contexto de trabalho, mesmo aquelas que reflectem uma dimensão mais subjectiva da actividade de trabalho, constitui uma das preocupações centrais do INSAT2010 e um ponto de ancoragem, permanente, na abordagem metodológica proposta.

\subsection{Incorporar a dimensão subjectiva dos efeitos do trabalho na saúde}

Tradicionalmente, os problemas de saúde no trabalho aparecem associados a um conjunto de patologias oficialmente reconhecidas como doenças profissionais exigindo, para isso, o estabelecimento de uma relação de causa-efeito entre a exposição a determinados riscos profissionais e o aparecimento da doença. Neste sentido, o objectivo consiste em delimitar a doença, definida a partir do diagnóstico médico que, frequentemente associada a um conceito de saúde clássico, ignora o estatuto de outros sinais ou queixas, quer se trate ou não de "sinais" patológicos. Ora, é frequentemente "com estes 'pequenos' problemas que os investigadores e técnicos em saúde no trabalho se vêem confrontados" (Gollac \& Volkoff, 2000, p. 21, tradução livre) e que o Inquérito INSAT procura integrar.

21 Já a versão INSAT2007 apelava para uma definição de saúde no trabalho mais abrangente e multidimensional, em que interagem as dimensões física, psicológica e social, e agora, nesta versão actualizada, a preocupação é a de dar mais visibilidade aos "pequenos" problemas de saúde, reforçando a noção de "bem-estar no trabalho" (Maggi, 2006).

22 Nesta perspectiva, o estudo da saúde no trabalho ao adoptar uma abordagem dirigida ao bem-estar, reveladora de relações menos visíveis entre a saúde e o trabalho, assume uma perspectiva mais centrada na pessoa, no que, aliás, Dejours (2009/1980) caracterizou como o "vivido subjectivo do trabalhador" (p.35) - revelador de novas relações, mais discretas, entre a saúde e o trabalho. As dimensões pessoal, interpessoal e transpessoal da actividade de trabalho (Clot \& Leplat, 2005) testemunham estes efeitos menos visíveis do trabalho na saúde e a necessidade de importar para o estudo da saúde ocupacional uma dimensão mais subjectiva, que facilita a identificação e a clarificação desses efeitos (nomeadamente, de carácter psicossocial, como o medo, a angústia, a irritabilidade ou a apatia).

$\mathrm{Na}$ verdade, uma orientação para o bem-estar coloca no centro da análise a preocupação com o sujeito que, ao desenvolver a sua actividade põe em aç̧ão diferentes dimensões do uso da saúde (Thébaud-Mony, 2010). Não se trata apenas do uso da dimensão física, da dimensão mental e mesmo da dimensão afectiva, mas de um uso de si (Schwartz, 1997). Nesta óptica, e retomando a dimensão subjectiva da saúde de 
Canguilhem (1999/1943) sentir-se de boa saúde traduz-se, assim, num poder agir sobre si e sobre o mundo que o rodeia (Clot, 1999), que se concretiza na forma como cada um gere a sua vida no trabalho e constrói a sua saúde.

Esta preocupação em caracterizar e avaliar os efeitos menos visíveis, mais discretos, da saúde constituiu desde o início um dos pontos de ancoragem do INSAT2007, que agora é reforçado. Isto é visível pela integração no INSAT2010 de um questionário de autoapreciação do estado de saúde - versão portuguesa do Perfil de Saúde de Nottingham, como foi já referido. A análise mais aprofundada destes indicadores permitirá destacar outras relações entre a saúde e o trabalho que, embora não retratem situações patológicas, perturbam o bem-estar dos trabalhadores e dificultam a sua vivência no dia-a-dia de trabalho.

\subsection{Facilitar a expressão e a explicitação do vivido no trabalho}

Outro dos pontos em que se sustenta a abordagem metodológica do INSAT prende-se com uma aplicação de "auto-preenchimento", susceptível de facilitar a expressão e a explicitação do vivido no trabalho. A estrutura do inquérito e o tipo de questões colocadas constituíram elementos promotores desta tomada de consciência e uma evolução progressiva quer na reflexão desenvolvida, quer nas declarações realizadas (Barros-Duarte, Cunha \& Lacomblez, 2007).

Assumindo uma perspectiva centrada na pessoa, o objectivo não é, de facto, o de confirmar ou infirmar o que dizem os trabalhadores: não é no confronto entre os conhecimentos dos investigadores e a experiência dos trabalhadores que se fundamenta esta orientação. Pelo contrário, a nossa experiência de análise neste domínio tem corroborado o facto de as declarações feitas pelos trabalhadores nem sempre serem explícitas e precisas, sendo desenvolvidas e enriquecidas pelo trabalho conduzido em comum com os investigadores - sem nunca esquecer os princípios teórico-metodológicos da ergonomia da actividade, isto é : o conhecimento real das situações de trabalho servirá, sempre, como pano de fundo para que o trabalho seja colocado em debate.

Ora, nem todos os aspectos do trabalho acabam por ser elegíveis neste debate : são sobretudo aqueles que se tornam experiência de sofrimento ou aqueles que interpelam particularmente o uso de si (Schwartz, 2000). Este uso não é somente heterodeterminado, mas é também o uso que cada um faz de si mesmo, na tentativa de um compromisso entre objectivos nem sempre facilmente conciliáveis e nem sempre fáceis de explicar. As declarações dos efeitos do trabalho na saúde acumulam os efeitos destes diversos usos de si mesmo.

No campo da saúde no trabalho, como realça Molinié (2010), “a expressão dos problemas de saúde pelas próprias pessoas é portanto uma questão central neste domínio que não é balizado pelas démarches diagnósticas e onde as maneiras de dizer mantém relações estreitas com as maneiras de viver a dor, ou a fadiga, ou seja, no sentido pleno do termo, com o que se experiencia" (p.70). O reconhecimento do vivido pelo trabalhador, muitas vezes traduzido pelas queixas e expressões de sofrimento, ganha um outro significado e um sentido coerente quando articulado com a expressão da actividade humana no trabalho.

Neste sentido, e de modo a facilitar a expressão e explicitação dos problemas de saúde no trabalho, foram introduzidas mudanças na operacionalização das questões colocadas 
no INSAT (organização dos itens, descrição dos itens, escalas de resposta, como já referido anteriormente), de modo a consolidar uma melhor compreensão das declarações proferidas e uma maior integração dos resultados do inquérito.

\subsection{Desenvolver uma estatística aberta}

30 Apesar das potencialidades estatísticas deste tipo de estudos, expressas na tentação de elaborar análises quantitativas globais e generalistas, Daubas-Letourneux (2004), Volkoff $(2002 ; 2005 ; 2010)$, entre outros autores, costumam realçar o quanto as interpretações dos resultados deverão ser acompanhadas por uma reflexão prudente e cuidadosa na sua compreensão, tendo em consideração a complexidade de que se revestem as relações entre saúde e trabalho.

31 Como já fora relatado na apresentação do INSAT2007 (Barros-Duarte, Cunha, Lacomblez, 2007), uma das preocupações presentes na formulação e concepção do inquérito, foi a de seleccionar questões que tivessem sido analisadas e debatidas nos estudos de terreno. Ora, se isto facilita a expressão e a explicitação do vivido pelo trabalhador, não é óbvio que tal seja suficiente para garantir que as análises estatísticas sejam reveladoras de relações entre saúde e trabalho.

De facto, o recurso às pesquisas qualitativas assume uma importância crucial nas análises dos resultados estatísticos (Derriennic, Touranchet, \& Volkoff, 1996), nomeadamente os estudos de terreno que, recorrendo à análise da actividade de trabalho, permitem completar, enriquecer e confirmar os resultados obtidos (Volkoff, 1998).

33 Na verdade, e a propósito da combinação entre os métodos quantitativos e qualitativos, Volkoff (2005) vai mais além nesta reflexão e, recuperando a ideia de saberes-fazer de prudência proposto por Cru \& Dejours (1983), salienta o quanto as análises estatísticas no domínio da saúde no trabalho devem ser prudentes e cautelosas. Procura-se, desta forma,

\footnotetext{
“apelar não apenas a uma reflexão sobre a metodologia utilizada mas, igualmente, sobre a própria realidade estudada (o trabalho e as implicações para a saúde). Nesta perspectiva, mais do que explicar a fiabilidade dos números obtidos e os resultados estatisticamente significativos, assume-se uma démarche de quantificação que designamos de compreensiva" (Volkoff, 2005, p. 18).
}

34 Assumir uma abordagem metodológica da saúde no trabalho "mais compreensiva do que explicativa", significa que se procura escutar o ponto de vista dos trabalhadores, entender e compreender a sua percepção do impacto do trabalho na saúde (Honoré, 2002), promovendo uma orientação mais atenta às declarações que enunciam.

35 A orientação metodológica incorporada no INSAT traduz-se, deste modo, numa avaliação das consequências do trabalho na saúde e no bem-estar centradas nas declarações dos trabalhadores, mas sempre sustentadas em registos do terreno. Neste sentido, a interpretação das análises estatísticas deve ser desenvolvida e orientada em função dos objectivos e necessidades específicas de cada estudo.

A esta "prudência" estatística, em oposição à autoridade e fiabilidade inquestionável de muitas análises estatísticas, Volkoff (2010) designou de "estatística aberta", atribuindolhe três directrizes fundamentais : (i) Assegurar permanentemente uma análise atenta e 
um esforço constante de explicitação dos dados estatísticos. Quer na "cozinha estatística" (Volkoff, 2010, p. 8, tradução livre) preparatória, quer nas interrogações face aos resultados estatísticos obtidos, é importante prever espaços de debate interdisciplinar cruzado, de modo a que os resultados obtidos tenham sempre como objectivo a compreensão das realidades estudadas e não a produção de resultados estatísticos inquestionáveis; (ii) 0 que conduz, igualmente, a flexibilizar a ortodoxia estatística, deixando algum espaço de liberdade ao uso dos testes de significância e dos intervalos de confiança, de modo a não correr o risco de negligenciar algum resultado estatístico singular que, por não "ser significativo", não possa ser analisado e interpretado à luz de outros conhecimentos; (iii) E, neste sentido, manter a coerência com outros conhecimentos construídos a partir de diversos métodos e níveis de análise, criando condições para que a abordagem metodológica quantitativa possa encontrar o seu espaço de análise e investigação em abordagens mais "compreensivas" do que "explicativas".

\section{Conclusões}

Já intencionalizado na concepção da primeira versão do INSAT, o conceito de saúde no trabalho, integrando a avaliação dos seus aspectos patológicos (grandes patologias profissionais, tradicionalmente designadas de doenças profissionais), mas também dos seus aspectos ditos infra-patológicos (traduzidos nas queixas e no vivido) é, agora, reforçado, no INSAT2010, pela ideia de que a construção da saúde reflecte uma vivência pessoal e subjectiva, na maior parte das vezes, ainda pouco visível. Neste sentido, a dimensão pessoal constitui um indicador de excelência, revelador de uma abordagem da saúde mais próxima do conceito de bem-estar, ou seja, dependente do contexto e da singularidade com que são vividas as relações entre saúde e trabalho.

É nesta perspectiva que o INSAT2010 procura ultrapassar as tradicionais avaliações do impacto dos riscos na saúde e na segurança - a que Annie Thébaud-Mony (2010) se referiu como a concepção dominante dos riscos - reforçando uma abordagem mais centrada no vivido pelo trabalhador e, portanto, alusiva a um conceito de saúde e bemestar subjectivo, multidimensional e dinâmico. Ao enfatizar a análise das relações entre saúde e trabalho na actividade humana e no vivido subjectivo emergem outras questões que, no contexto da saúde, procuram suscitar novas relações, novos desafios, nomeadamente : as questões de género em saúde no trabalho (Vogel, 2002), o stress e os riscos psicossociais no trabalho (Thébaud-Mony \& Robatel, 2009).

O interesse demonstrado por diferentes tipos de interlocutores na utilização do INSAT sindicatos, empregadores, médicos do trabalho - coloca o desafio de poder partilhar experiências de utilização do instrumento, de diferentes pontos de vista, em diferentes contextos e situações de trabalho. A construção de uma plataforma de apresentação dos resultados, e mesmo a possibilidade de informatização do instrumento, criam oportunidades de levar a cabo um estudo comparativo dos dados analisados e o alargamento da rede de investigadores do INSAT.

Contudo, consideramos fundamental, por um lado, sensibilizar os utilizadores do INSAT para a sua metodologia de aplicação, mantendo um olhar atento relativamente aos usos possíveis do instrumento e, por outro lado, realçar a permanente abertura ao ponto de vista e à experiência desenvolvida por aqueles que o utilizam no âmbito das suas pesquisas e intervenções. 
41 Neste sentido, ao reconhecer-se o INSAT como um instrumento "vivo", sujeito a alterações ao longo do tempo, demarcamo-nos de uma óptica "determinista e [submetida] ao desafio da generalização" (Davezies, 1998, p. 174, tradução livre), amplificando as capacidades de compreender e de agir no domínio da saúde no trabalho (Volkoff, 2010).

\section{BIBLIOGRAFIA}

Barros-Duarte, C., Cunha, L. \& Lacomblez, M. (2007). INSAT : uma proposta metodológica para análise dos efeitos das condições de trabalho sobre a saúde. Laboreal, 3, (2), 54-62, http:// laboreal.up.pt/revista/artigo.php?id=37t45nSU547112311:499682571

Canguilhem, G. (1999/1943). Le normal et le pathologique ( $8^{\mathrm{e}}$ éd.). Paris : PUF. (Obra original publicada em 1943).

Cassou, B., Buisset, C., Brugère, D., Davezies, P., Derriennic, F., Desplanques, G., Laville, A., Marquié, J-C., Touranchet, A., \& Volkoff, S. (2001). Travail, Santé, Vieillissement : relations et évolutions. Toulouse : Éditions Octarès.

Clot, Y. \& Leplat, J. (2005). La méthode clinique en ergonomie et en psychologie du travail. Le Travail Humain, 4 (68), 289-316.

Clot, Y. (1999). Souffrance au travail, amputation du pouvoir d'agir, défenses et ripostes.

Communication présentée au Colloque de Namur. Namur, France, novembre.

Cru, D., \& Dejours, C. (1983). Les savoirs-faire de prudence dans les métiers du bâtiment. Les cahiers médico-sociaux, 3, 239-247.

Cunha, L., Nogueira, S. \& Lacomblez, M. (forthcoming). Beyond a man's world : Methodological contributions of considering gender when studying bus drivers' activity. Journal.

Daubas-Letourneux, V. (2004). Accidents du travail : des accidents de parcours ? Regards croisés sur le (re)construction de la santé et sur le parcours professionnel de victimes d'accidents du travail. Actes du Séminaire Vieillissement et Travail CRÉAPT-EPHE (pp. 37-45). Paris : Centre d'études de l'emploi.

Davezies, P. (1998). Recherche en ergonomie et santé. Actes du Colloque Recherche et Ergonomie (pp. 173-176). Toulouse.

Davezies, P. (2009). Dérèglements organisationnels, santé, pouvoir d'agir. Retirado em Julho, 22, 2010 de http://philippe.davezies.free.fr/download/down/ Souffrance\%20au\%20travail\%20pour\%20Pratiques.pdf.

Dejours, C. (2009/1980). Insatisfaction au travail et souffrance mentale. In A. Thébaud-Mony \& N. Robatel (Orgs.), Problèmes politiques et sociaux, 965, 35-49.

Derriennic, F., Touranchet, A. \& Volkoff, S. (1996). Age, travail, santé. Paris : INSERM

Gollac, M. \& Volkoff, S. (2000). Les conditions de travail. Paris : Éditions La Découverte.

Honoré, B. (2002). A Saúde em Projecto. Loures : Lusociência. 
Maggi, B. (2006). Bem-estar. Laboreal, 2, (1), 62-63, http://laboreal.up.pt/revista/artigo.php? id $=48 u 56$ oTV $6582234: 8365549762$

Molinié, A-F. (2010). Queixa, Laboreal, 6, (1), 69-71, http://laboreal.up.pt/media/artigos/ 300/69-71.pdf

Molinié, A-F., \& Volkoff, S. (2002). La démographie du travail pour anticiper le vieillissement. Paris: ANACT.

Quéinnec, Y. (2007). Horário. Laboreal, 3, (2), 90-91. http://laboreal.up.pt/revista/artigo.php? $\mathrm{id}=48 \mathrm{u} 56 \mathrm{oTV} 6582234234335473732$

Schwartz, Y. (1997) (Dir.). Reconnaissance du travail. Pour une approche ergologique. Paris : PUF.

Schwartz, Y. (2000). Trabalho e uso de si. Pro-posições 1, 5 (32), 34-49.

Thébaud-Mony, A. \& Robatel, N. (Orgs.) (2009). Stress et risques psychosociaux. Problèmes politiques et sociaux, 965 .

Thébaud-Mony, A. (2010). Riscos, Laboreal, 6, (1), 72-73, http://laboreal.up.pt/media/artigos/ 301/72-73.pdf

VISAT (1994). Project VISAT : Étude longitudinal sur la relation Vieillissement, Santé, \& Astreintes de Travail. Toulouse : CNRS

Vogel, L. (2002). La dimension de genre en santé au travail, BTS, 18, 14-19.

Volkoff, S. (1998). Représentativité, significativité, causalité : l'ergonomie au contact des méthodes épidémiologiques. In M-F. Dessaigne \& I. Gaillard (Eds.), Des évolutions en ergonomie... (pp. 225-236). Toulouse : Éditions Octarès.

Volkoff, S. (2002). Des comptes à rendre : usages des analyses quantitatives en santé au travail pour l'ergonomie. Noisy-le-Grand : Centre d'Etudes de l'Emploi. www.eurofound.europa.eu/ewco/ surveys/EWCS2005/index.htm.

Volkoff, S. (2005) L'ergonomie et les chiffres de la santé au travail : ressources, tensions et pièges. Toulouse : Éditions Octarès.

Volkoff, S. (2010). Statistiques "ouvertes" et ergonomie "myope" : combiner les niveaux d'analyse en santé au travail. Sciences Sociales et Santé, 28, 2, 11-30.

\section{NOTAS}

1. Falamos designadamente dos inquéritos da Fundação de Dublin: European Foundation for the Improvement of Living and Working Conditions (2001). Third European survey on working conditions 2000. Luxembourg: Office for Official Publications of the European Communities.

European Foundation for the Improvement of Living and Working Conditions (2007). Fourth European survey on working conditions 2005. Luxembourg: Office for Official Publications of the European Communities.

2. Centro de Estudos da Saúde do Trabalhador e Ecologia Humana, Escola Nacional de Saúde Pública Sérgio Arouca, Fundação Oswaldo Cruz, Rio de Janeiro.

3. Versão portuguesa, adaptada pela Faculdade de Economia da Universidade de Coimbra, Centro de estudos e Investigação em Saúde, 1997, sob responsabilidade do Prof. Doutor Pedro Lopes Ferreira.

4. SIT (2001). Inquérito SIT - Saúde, Idade e Trabalho. Porto: Centro de Psicologia da UP \& IDICT. 


\section{RESUMOS}

O INSAT enquanto instrumento mediador das declarações do impacto do trabalho na saúde conta já com uma história que aqui procuraremos desvelar, referindo: os sectores de actividade considerados nas análises com este instrumento; os problemas encontrados em alguns desses contextos e situações de trabalho; os argumentos que justificam um estudo mais preciso de outras relações entre as variáveis contempladas no inquérito.

o retrato e a interpretação da história do INSAT contribuem finalmente para o seu reconhecimento como um instrumento "vivo", agora reformulado, tendo em conta as evoluções do trabalho, bem como as reflexões produzidas a partir da sua utilização.

El INSAT es un instrumento mediador de las declaraciones del impacto que tiene el trabajo sobre la salud, contando con una historia que pretendemos desvelar. Para ello, nos referiremos a los sectores de actividad, considerados en análisis previamente hechos con el instrumento ; a los problemas encontrados en algunos de estos contextos y situaciones de trabajo; y a los argumentos, que justifican un estudio más detallado de otras relaciones entre variables contempladas en la encuesta.

El retrato y la interpretación de la historia del INSTAT contribuyen, finalmente, a su reconocimiento como un instrumento "vivo", ahora reformulado, teniendo en cuenta a las evoluciones del trabajo, así como a las reflexiones producidas como consecuencia de su utilización.

L'INSAT, en tant qu'instrument de médiation des déclarations concernant l'impact du travail sur la santé, a déjà une histoire que nous cherchons à systématiser et analyser ici, en reprenant : les secteurs d'activité couverts par les études; les difficultés rencontrées, dans certains de ces contextes et situations de travail ; les arguments qui justifient une approche plus précise d'autres relations entre les variables traitées dans l'enquête.

Le rapport et l'interprétation de l'histoire de l'INSAT contribuent finalement à la valorisation d'un instrument "vivant ", qui a été reformulé en tenant compte des évolutions du travail, ainsi que des réflexions produites par son utilisation.

The INSAT, as an instrument that mediates the statements of the work's impact on heath has already a history that we will try to unveil. For that purpose we will refer to the activity sectors considered in previous analyses conducted with this instrument; to the problems found in some of these contexts and work situations; to the arguments that justify a more precise study of other relationships between the variables considered in the inquiry. Finally, the portrait and the interpretation of INSAT's history contribute to its acknowledgement as a "living" instrument, now reformulated considering the evolutions of work, as well as the reflections elicited by its use.

\section{ÍNDICE}

Palabras claves: Declaraciones, experiencia de trabajo, salud, bienestar, estadística abierta

Keywords: statements, work experience, health; well-being, open statistics

Mots-clés: déclarations, expérience de travail, santé, bien-être, statistique ouverte

Palavras-chave: Declarações, experiência de trabalho, saúde, bem-estar, estatística aberta 


\section{AUTORES}

\section{CARLA BARROS-DUARTE}

Universidade Fernando Pessoa , Praça 9 de Abril, nº 349 , 4249-004 Porto

cbarros@ufp.edu.pt

\section{LILIANA CUNHA}

Universidade Católica Portuguesa, Rua Diogo Botelho, 1327, 4169-005 Porto e Centro de Psicologia da Universidade do Porto, Rua do Dr. Manuel Pereira da Silva , 4200-392 Porto

lcunha@porto.ucp.pt 